\title{
Comunicación del cambio climático y generación de capacidades adaptativas entre los agricultores del trópico subhúmedo
}

\author{
Lorena Casanova-Pérez ${ }^{1 \S}$ \\ Juan Pablo Martínez-Dávila² \\ Florencia García-Alonso ${ }^{1}$ \\ Emigdio de la Cruz-de la Cruz ${ }^{1}$ \\ ${ }^{1}$ Universidad Tecnológica de la Huasteca Hidalguense. Carretera Huejutla-Chalahuiyapa s/n, Colonia \\ Tepoxteco, Huejutla, Hidalgo, México. CP. 43000. (florencia.garcia@uthh.edu.mx; \\ emigdiodelacruz@uthh.edu.mx). ${ }^{2}$ Programa Agroecosistemas Tropicales-Colegio de Postgraduados. \\ Carretera Xalapa-Veracruz km 85.5, Predio Tepetates, Manlio Fabio Altamirano, Veracruz. CP. 91690. \\ (jpmartin@colpos.mx). \\ ${ }^{\S}$ Autor para correspondencia: lorena.casanova@uthh.edu.mx.
}

\section{Resumen}

Este ensayo analiza la importancia de la comunicación del cambio climático en un contexto local específico poniendo énfasis en la semántica usada por los medios masivos influenciada por un discurso científico-técnico, asimismo, reflexiona sobre las oportunidades para hacer que dicha comunicación sea efectiva y convierta al cambio climático en un tema de comunicación social. Lo anterior requirió fundamentalmente de una revisión documental sobre el papel de los medios masivos en la comunicación del cambio climático y de los principios de la teoría Luhmanniana, así mismo, del uso de información generada previamente a través de una encuesta y entrevistas a profundidad. Los resultados muestran que la compresión del significado del cambio climático y sus impactos en la agricultura en el mediano y largo plazo requiere considerar la semántica como una construcción colectiva utilizada en las operaciones comunicativas por los productores y sus familias en el contexto local, esto posibilitará la generación de capacidades adaptativas y por ende, el diseño e implementación de acciones proactivas para la adaptación de la agricultura del trópico subhúmedo mexicano ante el cambio climático.

Palabras clave: adaptación, agricultura, semántica.

Recibido: junio de 2019

Aceptado: agosto de 2019 
El cambio climático es un fenómeno que en aproximadamente dos décadas y media de su inclusión como un tema en los principales medios de comunicación parece haberse convertido en una tema protagónico y recurrente entre la población, lo cual debiera estar influenciado la actitud y el comportamiento de esta (González, 2012). En el continuum de realidad, lo anterior se expresaría en una serie de acciones de mitigación y adaptación ante dicho fenómeno (Weingart et al., 2000). En otras palabras, la población debería tener un conocimiento general de sus efectos en su vida cotidiana y en el desarrollo de sus actividades productivas conduciéndolos a un mayor compromiso y participación para lidiar con los efectos reales.

Potenciales e inesperados de este fenómeno, sobre todo, en el mediano y largo plazo (O'Neill et al., 2013). En contrasentido, investigaciones recientes enfocadas particularmente a la comunicación del cambio climático desde los medios masivos evidencian que éste fenómeno ha dejado de ser relevante para la población, evidencia de esto son la falta de propuestas o acciones para contrarrestar sus impactos. Newig (2011) menciona que este hecho está relacionado a la controversia científica entre los climatólogos preocupados por el fenómeno versus los escépticos del clima, situación que ha generado incertidumbre entre la población sobre su existencia real.

Así, se pasó de un discurso donde el cambio climático era planteado como el meta problema omnipresente de la humanidad, a ser posteriormente un problema de regulación política normal y rutinaria, disminuyendo la percepción de su peligrosidad y urgente necesidad de establecer estrategias para enfrentar sus impactos (Weingart, 2000). En regiones de Europa y Norteamérica, el cambio climático es aún un tema en debate, al cual subyace la incertidumbre y la controversia (Wibeck, 2013).

En México, los estudios sobre la comunicación del cambio climático se consideran de carácter emergente, particularmente la comprensión del papel que juegan la televisión y la radio como los principales medios de comunicación al hacer disponible la información rápidamente a una audiencia numerosa (González, 2012). Al respecto, la ENDUTIH (2019) expone que, 2018; 95.4\% de los hogares mexicanos contaba con televisión digital, en contraste con 52.9\% de los hogares con acceso a internet mediante conexión fija o móvil. Esta encuesta reveló también que el uso de internet es un fenómeno urbano, ya que $73.1 \%$ del total de la población en las ciudades son usuarios de este servicio, mientras que en las zonas rurales únicamente es de $40.6 \%$.

Tanto la televisión como el internet son medios que han registrado un desarrollo impresionante en los últimos años debido a una serie de inventos tecnológicos que establecieron nuevas dimensiones para la distribución de la comunicación (Osorio, 2011). Lo anterior, es un tema importante puesto que mucho de lo que sabemos acerca de este fenómeno ha sido a través de ellos. Esto requiere una reflexión profunda de los motivos y metas de la comunicación, cómo son construidos los mensajes y cómo deberían de serlo, en qué contexto y si estos realmente son comprendidos (Wibeck, 2013; Leombruni, 2015).

Hasta ahora los hallazgos del quehacer investigativo en otras latitudes indican que las estrategias de comunicación sobre el cambio climático han fallado y nos enfrentamos a una audiencia indiferente aparentemente (Ockwell et al., 2009). Por lo tanto, son necesarias investigaciones que permitan generar conocimiento particularmente en relación con el fortalecimiento de capacidades adaptativas (Adger et al., 2005). 
Conocimiento que pudiera ser eventualmente incorporado en el diseño de estrategias de comunicación desde instituciones del sector público y privado que tengan interés real en coadyuvar los esfuerzos encaminados al desarrollo de capacidades de la población. Particularmente de los agricultores para establecer prácticas que les ayuden a mitigar y adaptarse al cambio climático en el mediano y largo plazo. En consecuencia, este documento tiene como propósito dilucidar el rol que juegan los medios de comunicación y la semántica usada en su discurso en la percepción del cambio climático entre los agricultores en parte del trópico subhúmedo mexicano; asimismo, reflexionar sobre las oportunidades para hacer dicha comunicación efectiva que convierta al cambio climático en un tema de comunicación social y se briden condiciones de posibilidad para generar entre los agricultores capacidades adaptativas ante dicho fenómeno.

\section{El cambio climático y los medios de comunicación: una primera aproximación}

El cambio climático es un tema cotidiano en los medios de comunicación, los cuales informan sobre los avances científicos, los desacuerdos entre la comunidad internacional y las políticas de respuesta institucional (González, 2012). Sin embargo, su comunicación se ha caracterizado por un discurso estandarizado que se impulsó desde el ámbito científico, un discurso con una semántica común que facilitó el intercambio de conocimientos propiciando un entendimiento mayor entre investigadores alrededor del mundo (McNaught et al., 2014).

De ese modo, los conocimientos generados robustecieron y permitieron posicionar a la teoría del cambio climático antropogénico como el paradigma dominante (IPCC, 2013), este avance contrasta con la percepción y comprensión del fenómeno en el nivel local en varias regiones del mundo (Sterman, 2011; McNaught et al., 2014). Es decir, existe evidencia empírica que permite inferir la existencia de una comprensión raquítica sobre el cambio climático en parte de la sociedad (Leombruni, 2015).

Esto puede asociarse al uso de conceptos que no son parte de la semántica usada en las operaciones comunicativas de las personas que viven en ciertas regiones/localidades Esto es porque la semántica como el lenguaje no lo inventa alguien en particular, sino que, es una construcción colectiva de la comunidad de hablantes (Lavid, 2003; Rahman, 2013).

Una comunidad de hablantes que propone de una generación a otra, inter-generacionalmente una determinada semántica que se va reinterpretando, asumiendo críticamente y re-adecuando a las nuevas condiciones. Lo anterior, debe ser un punto de debate entre los tomadores de decisiones y su estrategias de comunicación no solo en el nivel central, sino también en las diferentes estructuras de administración que en caso de México se refieren a los niveles estatales y municipales, de tal modo que esto ayude a que los tomadores de decisiones entiendan la vulnerabilidad y el riesgo en el nivel local tal como lo menciona y actúen en consecuencia, en estrategias para desarrollar capacidades adaptativas entre los miembros de la sociedad.

Bajo este contexto, se plantea que hasta ahora la comunicación del cambio climático desde los medios no ha considerado las diferentes audiencias relacionadas a características como: edad, sexo, nivel escolar, actividad productiva, contexto cultural y tampoco, la semántica que subyace a su comunicación (Buys et al., 2012; Schweizer et al., 2013; Wibeck 2014; Leombruni, 2015). 
Esto posibilita, por ejemplo, que se generen diversos significados para quienes se dedican a la ciencia, la política, la educación, la agricultura, o para quienes viven en ciudades o zonas rurales. Esto sucede porque no existe una traducción semántica del discurso del cambio climático en el lenguaje local (Lata y Nunn, 2012; Rahman, 2013). Estas audiencias diferentes deben ser consideradas en cualquier estrategia comunicativa, su omisión ha traído como resultado: indiferencia, escepticismo e invisibilidad del fenómeno (Wibeck, 2013; Leombruni, 2015). En el caso de la indiferencia ante el cambio climático, esta se deriva de una percepción de normalidad entre la población que genera y fortalece una especie de anomia social en cuanto al compromiso de participar en acciones encaminadas a su adaptación y mitigación (González, 2012). En cuanto a su escepticismo, ejemplo de ello son los hallazgos de los cuales muestran que en Estados Unidos de América 75\% de los demócratas creía en la existencia de evidencia sólida del calentamiento global, comparado con $35 \%$ de republicanos y $53 \%$ de independientes, percepción relacionada a su posición ideológica-económica (Maeseele y Pepermans, 2017).

En cuanto a la invisibilidad del cambio climático, O’Neill et al. (2013), explica que las personas frecuentemente lo perciben como distante en cuanto a su experiencia diaria, o creen que las variaciones del clima son temporales y éste regresará a sus condiciones normales en el futuro. Esta idea ha sido reforzada cuando los medios masivos de comunicación asocian la presencia del cambio climático a eventos catastróficos que suceden en un espacio lejano y tiempo en particular: deshielo de los casquetes polares, sequías extremas en el África Subsahariana, etcétera (Nielsen y Reenberg, 2010; Newig, 2011).

Al respecto, es importante reconocer que el cambio climático no es un tema fácil de abordar ya que su propia complejidad dificulta su comprensión en la sociedad (Nerlich et al., 2010; Raymond and Spoehr, 2013; Caglea y Tillery, 2015), así como, identificar los límites de la percepción humana y los errores de comunicación por parte de los científicos del clima (Moser y Dilling, 2004). Adicionalmente, se recomienda que la comunicación del cambio climático en los medios debería considerar información tanto cuantitativa como cualitativa, esta última referida a las implicaciones culturales y sociales que este fenómeno tiene en los contextos locales (Newig, 2011).

Este es un gran reto para los comunicadores de los medios masivos, quienes no debe caer en la simplificación del fenómeno no obstante la complejidad que le es inherente (O’Neill et al., 2013) ya que existe la posibilidad de que los malentendidos en la audiencia puedan tener un impacto negativo en los esfuerzos encaminados a generar capacidades adaptativas en la sociedad para su mitigación y adaptación.

Así, en los últimos cinco años, la comunicación del cambio climático se ha convertido en un tema de investigación emergente que busca convertirle en un tema más comprensible (Newig, 2011). Autores como Eskjær (2012); Eaking et al. (2014); Fernández-Llamazares et al. (2015); evidencian la necesidad de comprender por qué la cobertura de los medios de comunicación dedicados al cambio climático está decreciendo, cuales son los motivos por los cuales la audiencia, particularmente en los países de occidente, está perdiendo el interés sobre sus efectos y está menos interesada por aprender al respecto. Esto es nodal, ya que puede convertirse en un límite para el logro del compromiso público y apoyo para lidiar con los impactos de este fenómeno (Wibeck, 2013). 


\section{Medios de comunicación, percepción del cambio climático y agricultura: hallazgos preliminares en el trópico subhúmedo mexicano}

La comunicación del cambio climático desde los medios debe considerar que es un fenómeno de carácter global con efectos locales diferenciados (Casanova-Pérez et al., 2016), por lo cual requiere una comprensión del fenómeno y de las características del contexto local/regional, de tal modo que los mensajes sean apropiados y significativos (Leal-Filho, 2009; McNaught et al., 2014). Sobre todo, cuando se trata de una región donde sus habitantes tienen como actividad productiva principal a la agricultura y han construido una semántica particular que expresa una forma de describir el mundo y una manera específica en que los productores agrícolas se comunican, la cual es fundamentalmente verbal y se realiza cara a cara (Altieri y Nicholls, 2008; Howe et al., 2013; McNaught et al., 2014).

Así, lograr la comunicación adecuada del cambio climático es fundamental para la adaptación, ya que este proceso es primeramente un acto de manejo del riesgo (Newig, 2011; IPCC, 2013). Pero ¿Qué sucede en un continuum de realidad particular? La respuesta a este cuestionamiento fue estructurada a partir de la información generada por una investigación previa realizada en las localidades de El Limón, Angostillo, Xocotitla y Rancho Nuevo del municipio de Paso de Ovejas, Veracruz, región que es parte del trópico subhúmedo mexicano (INEGI, 2010). Esta investigación involucró una encuesta con 135 productores participantes en un rango de edad de 20 a 80 años. La encuesta se realizó con base en una muestra estadística estratificada por edad y por localidad.

La muestra se obtuvo de un marco muestral elaborado con información del registro agrario nacional, el Instituto Mexicano del Seguro Social y los presidentes de los comisariados ejidales. Los datos fueron sistematizados y analizados de forma descriptiva con el programa Statistica 7.1 Tulsa, OK, USA) (Casanova-Pérez, 2015). Los resultados obtenidos muestran que de los productores encuestados en estas cuatro localidades únicamente $41.7 \%$ dijo haber escuchado hablar del cambio climático. Sin embargo, de estos productores solo $26.6 \%$ tuvo una idea parcial del fenómeno, mientras que $73.4 \%$ mencionó que no lo comprendía.

De los entrevistados que tuvieron una comprensión parcial del fenómeno, solo 19.6\% manifestó que el cambio climático puede afectar su quehacer agrícola. Las repuestas ante la pregunta de por qué está sucediendo el cambio climático aluden a que 'ya no hay árboles', 'hay secamiento de lagunas', 'a la deforestación y al uso de líquidos (agroquímicos)'. Estas respuestas indican la comprensión mínima que tienen los productores del fenómeno y por lo tanto la imposibilidad de conocer sus implicaciones en su quehacer agrícola, sobre todo, en el mediano y largo plazo.

Dicha información fue obtenida $90 \%$ a través de la televisión y $10 \%$ por la televisión y la radio. Por último, del total de productores solo $2.7 \%$ indicó haber conversado con sus compañeros al respecto, es decir, el cambio climático no es un tema recurrente en sus conversaciones (CasanovaPérez, 2015). Ante esta evidencia, se plantea que, en el área de estudio, el cambio climático aún no es percibido por los agricultores como una amenaza real ya que la modificación de los patrones de precipitación y temperatura ha sido vista como parte de la variabilidad natural.

La adaptación a esta situación se ha limitado básicamente al cambio en las fechas de siembra y al intercambio/renta de forraje y acceso al agua para el ganado bovino. Una situación reforzada por una comunicación ineficaz y un apoyo gubernamental inexistente que ha reducido las posibilidades 
de desarrollar estrategias adaptativas de carácter proactivo (Campos et al., 2013). En este mismo sentido, Soares y García (2013) arguyen que el Estado mexicano y sus instituciones tienen un gran reto en cuanto al fortalecimiento de las capacidades de adaptación de los grupos más vulnerables particularmente aquellos dedicados a la actividad agrícola en zonas históricamente marginadas.

A esta idea, se suman los resultados obtenidos por la encuesta realizada por el Consejo Nacional de Ciencia y Tecnología (CONACYT) y el Instituto Nacional de Estadística, Geografía e Informática (INEGI) sobre la percepción pública de la ciencia y la tecnología en México, donde un apartado fue destinado a conocer la percepción de la población sobre el cambio climático. Los resultados muestran que solo un $33 \%$ de la población de la población urbana radicada en la Ciudad de México y en las ocho ciudades más importantes del país entendía la naturaleza del fenómeno, este reducido entendimiento es aún menor en las zonas rurales donde la agricultura tiene un papel preponderante como medio de vida de la población.

Ante estos hechos, se propone que la prioridad de la comunicación del cambio climático en el contexto local debe ser que éste se convierta en un tema social y con el tiempo pase a formar parte de su memoria colectiva. Concepto definido por un conjunto de recuerdos y expectativas del sistema de comunicación, es decir, del conjunto de temas de los que se habla en una sociedad. Esta memoria permite que los agricultores cuenten con información valiosa, seleccionada y actualizada para situaciones contingentes como lo es el cambio climático.

Este planteamiento visto desde las localidades ubicadas en el trópico subhúmedo mexicano significa lograr la comprensión de este fenómeno por parte de los agricultores, de sus impactos en su quehacer agrícola en las próximas décadas y de ese modo tomar decisiones al respecto desde sus recursos disponibles (Boon, 2014). Al respecto, argumenta que la comunicación como comprensión permite que se generen condiciones de posibilidad que conduzcan a procesos reflexivos que permitan pasar a la acción.

¿Cuáles son las condiciones que se requieren para que el cambio climático sea comprendido? En primer lugar; es necesario, reconocer que existen diferencias sustanciales entre quienes detentan el conocimiento (científicos), los encargados de comunicar dicho conocimiento (comunicadores) y los agricultores. En segundo lugar, hacer una revisión de la forma como se ha dado esta comunicación. González (2012) plantea que las estrategias de comunicación del cambio climático en medios de comunicación masiva han sido basadas en la teoría del déficit de conocimiento.

En México como otras partes del mundo, esta teoría de carácter tecnocrático ha sido el fundamento del modelo de comunicación utilizado por los medios masivos, el cual consiste en tres elementos (emisor-mensaje-receptor). Desde este modelo, el emisor está al inicio del proceso comunicativo, como el que determina los contenidos de este y las ideas que se quiere comunicar, mientras que el destinatario está al final, como simple receptor, recibiendo el mensaje (Ibarra, 2001).

Así, los mensajes son enviados de una forma lineal desde quien posee los conocimientos hacia quienes tienen déficit de estos (Newig, 2011). Este modelo tiene sus bases en el modelo de Shannon, el cual presenta a la comunicación como un sistema de transmisión discreta de mensajes que está organizado en función de una fuente, un mensaje, un canal y un receptor y cuyo funcionamiento es lineal (Pignouli, 2013). Tal como sucede con otros modelos como el contextual, la pericia/legal y el de participación pública (Sagástegui, 2015). 
Con base en lo anterior, la comunicación del cambio climático implica ir más allá de la interpretación lineal de la producción y diseminación de la información (Eakin et al., 2014). Es necesario encontrar modelos que consideren a la comunicación basada en el diálogo y la comprensión con base en cierto contexto cultural (Nerlich et al. 2010). En este sentido, a continuación, se expone los principios de un modelo alternativo de comunicación basado en la teoría de sistemas sociales autopoiéticos (TSSA) de Niklas Luhmann.

\section{La comunicación como comprensión en la agricultura: modelo de comunicación alternativo desde la teoría Luhmanniana}

La comunicación del cambio climático por sí misma es insuficiente para motivar acciones de adaptación y mitigación, sin embargo, una comunicación adecuada puede facilitarlas (Eaking et $a l ., 2014)$. Esto es porque la comunicación de este fenómeno es parte de la comunicación del riesgo, aquella que tiene como fin que las personas estén informadas y puedan tomar decisiones con base en juicios sobre los riesgos que este fenómeno implica en su seguridad, salud y actividad productiva (Newig, 2011).

Por ello, uno de los temas relevantes es comprender el papel que juegan los medios masivos para hacer que la comunicación sobre el cambio climático se vuelva probable. Sin embargo, desde los datos empíricos mostrados con anterioridad, se infiere que el papel de los medios masivos en la comunicación del cambio climático, al menos en el trópico subhúmedo mexicano, ha sido limitado, porque su papel no debería radicar únicamente en que un agricultor haya escuchado hablar sobre el cambio climático, en otras palabras no basta con que un comentarista de televisión o radio lea y comente sobre un documento, un boletín, o brinde una noticia sobre el deshielo polar, los efectos de una sequía, un tornado o de una inundación grave (como fenómenos asociados al cambio climático).

Una adecuada comunicación del cambio climático debería generar entre los agricultores su propia conceptualización del fenómeno. Si los agricultores están informados en ese sentido, pueden comprometerse en acciones, estableciendo cambios que puedan facilitar el desarrollo de estrategias de mitigación y adaptación a mediano y largo plazo, incluso aquellas que implican el cambio de normas y valores. En ese sentido, es importante preguntarse ¿Cuáles son las condiciones que los comunicadores desde los medios de comunicación masiva deben considerar para que los productores del trópico subhúmedo mexicano comprendan el cambio climático?

En primer lugar, considerar la semántica de los relatos, las historias y todo tipo de narraciones por los lugareños (Wolf y Moser, 2011), así como, las conceptualizaciones que tienen sobre el clima y sus principales variables, todo ello como una forma de expresión de una forma particular de ver el mundo, incluida sus preocupaciones como agricultores (Baraldi, 1996; Fowler, 2008; plantea que la semántica también subyace al conocimiento tradicional que tiene la sociedad sobre sí misma y sobre su entorno, por tanto, debe considerársele como un patrimonio conceptual de la sociedad, son los temas sobre los cuales se comunica y las palabras usadas en ello. Se trata de conceptos e ideas por usar y eventualmente construir concepciones del mundo, tal como se expresa en el Cuadro 1. 


\begin{tabular}{cc}
$\begin{array}{l}\text { Cuadro 1. Diferenciación semántica de la lluvia en cuatro localidades de Paso de Ovejas versus } \\
\text { semántica científica. }\end{array}$ \\
\hline Semántica científica & Semántica en el contexto local \\
\hline Lluvias torrenciales & Aguacerones, torrentales \\
Lluvias aisladas & Lluvias manchoneadas \\
Lloviznas ligeras & Pelillo de gato \\
Lluvias ocasionales & Lluvias aventureras \\
Lluvia & Lluvia pausada, lluvia penetrante \\
\hline
\end{tabular}

Fuente: Casanova-Pérez (2015).

Lo antes expuesto es un ejemplo puntual sobre la semántica particular en una sociedad agrícola, la cual expresa una concepción del mundo particular que debe ser considerada por los estrategas de la comunicación. Otros aspectos para considerar, es que la semántica evoluciona y que en las sociedades rurales se alimenta de la comunicación oral y está vinculada a la memoria de las personas (Baraldi, 1996). Así se pasó del uso del concepto 'lloviznas frijoleras', hidrometeoro que proveía de humedad al suelo para el cultivo del frijol en el último cuatrimestre del año, a las lloviznas denominadas ‘pelillo de gato', construcción semántica que indica lloviznas ligeras o de poca intensidad.

Es decir, las estrategias de comunicación sobre los efectos del cambio climático deberán considerar la diferencia entre estos dos conceptos, diferencia que indica cambios en la intensidad del hidrometeoro en cuestión en el área de estudio, lo anterior favorecería la comprensión del cambio climático y de sus impactos en el comportamiento de las principales agroclimáticas y su relación con la agricultura.

En consecuencia, lo anterior requiere dejar a un lado el modelo de comunicación lineal basado en la teoría del déficit del conocimiento y anteponer la búsqueda de modelos cuyo enfoque permita anteponer a la comprensión como el fin último de la comunicación del cambio climático (Eakin et $a l ., 2014)$ en los cuales la semántica juega un papel fundamental. Es decir, superar el modelo de comunicación tradicional donde el emisor esta al inicio del proceso comunicativo y es quien determina los contenidos y lo que desea comunicar.

En tanto el receptor, el destinatario final, es un simple receptor. Lo que se requiere es que exista un proceso de comunicación real, donde la información tenga más probabilidades de ser comprendida, se debe situar en la realidad concreta del destinatario (Ibarra, 2001). Lo anterior, exige la identificación de modelos que consideren a la comunicación basada en el diálogo y la comprensión con base en cierto contexto cultural (Nerlich et al., 2010). Ante este contexto, resulta interesante dilucidar sobre las posibles aportaciones de un modelo de comunicación basado en la teoría Luhmanniana cuya principal premisa teórica es la comunicación como comprensión, la cual se hace probable con la ayuda de los medios masivos de comunicación facilitando su aceptación. 
Pero ¿cómo debe comunicarse el cambio climático tomando en cuenta la teoría Luhmanniana? En primer lugar, habría que considerar los hallazgos encontrados en el área de estudio, los cuales permiten inferir que la comunicación sobre el cambio climático, aunque ha sido un tema abordado recurrentemente por los medios masivos, no ha garantizado que los productores agrícolas de esta área hayan comprendido su origen e impactos. En otras palabras, para el diseño de estrategias de comunicación debe considerarse que lo informado sobre el cambio climático; a través, de los medios de comunicación, no será siempre lo mismo que el productor finalmente entendió (asumiéndose que hubo perdida de información) y comunicó a su familia y demás productores.

Una estrategia para reducir la brecha entre lo informado por los medios de comunicación y lo comprendido por los productores del área de estudio deberá considerar la importancia del uso de una semántica que corresponda a la audiencia a quien se dirige dicha estrategia de comunicación. Ejemplo de ello son las diferentes construcciones semánticas con relación a los tipos de precipitación, las cuales por si mismas aluden a cambios en la distribución y la intensidad de la lluvia y lloviznas.

Otra estrategia es asociar los impactos del cambio climático en el área de estudio con la actividad agrícola, en particular, lo relacionado a las pérdidas de ingresos originados temperaturas no óptimas, disminución de la precipitación, vientos intensos y estiajes prolongados. Es decir, comunicar sobre los efectos negativos en el desarrollo de sus cultivos y crianza del ganado (mayor presencia de plagas y enfermedades por las altas temperaturas, susceptibilidad a enfermedades de los cultivos por mala nutrición debido a falta de humedad en el suelo, pérdida de semilla).

Desde la TSSA, el éxito de la comunicación del cambio climático es que éste se convierta en un tema social, porque la redundancia en las operaciones comunicativas produce saber, ese saber es un conocimiento valioso que ayuda a tomar decisiones anticipadas, autónomas, etc. En otras palabras, el productor actúa en muchos de los casos de manera completamente rutinaria lo cual puede ser comprendido como una repetición de una decisión ya adoptada. Entonces, para que éste realice cambios requiere de información cuya previa valoración le permita tomar una decisión. Las decisiones son, por lo tanto, el resultado de la tematización de una contingencia (cambio climático).

Este modelo que toma a la comunicación como comprensión supondrá el reconocimiento de este fenómeno y la toma de conciencia de los riesgos que este implica para los agricultores en su quehacer productivo. Los resultados de la comunicación como comprensión brindarán condiciones de posibilidad para que los agricultores se comprometan con acciones reales y dirigidas en la adaptación ante ese fenómeno derivadas de estrategias autónomas o soportadas por agentes externo con relación a la adaptación del cambio climático, pero sobre todo, a un tipo de adaptación planeada en el largo plazo.

Asimismo, podrán a través de una participación ciudadana lograr la incorporación del cambio climático como un tema central en los planes de desarrollo, considerando a la agricultura como un sector vulnerable y responsable de la seguridad alimentaria de una región o país. En otras palabras, conformar una agenda política o el uso de los mismos medios de comunicación para comunicar su protesta social logrando con ello un apoyo social mayor en otros sectores de sociedad (Solís y Salvatierra, 2013). 
Esto en contracorriente a las políticas públicas del país que se caracterizan por su desarticulación (Barrasa, 2017) y por la necesidad de una coordinación intersectorial e interinstitucional (transversalidad), impulsando con ellos estrategias para impulsar la economía del cambio climático, educación, capacitación e investigación, así como la de información y de comunicación del fenómeno que conduzcan a estrategias de adaptación (Olmos et al., 2013; Soares y García, 2014).

Finalmente, es necesario reiterar que la comunicación del cambio climático a los agricultores a través de los medios masivos es una estrategia que puede coadyuvar a la adaptación y la mitigación de este fenómeno: manejo de cultivos, manejo del suelo, riego, diversificación del ingreso, etc. (Safi et al., 2012; Esham y Garforth, 2013), llevarla a cabo requiere de la colaboración entre científicos del clima, investigadores dedicados a las ciencias sociales, entre ellos, los estudiosos de la comunicación bajo un enfoque multi e interdisciplinario.

Este conocimiento conjugado con una política de Estado, una participación interesada entre quienes detentan la posesión de los medios de comunicación masiva y el fortalecimiento de medios al servicio público, brindarán condiciones para el desarrollo de capacidades adaptativas entre los productores con el fin de lidiar con los efectos del cambio climático.

\section{Conclusión}

La tematización del cambio climático en los medios de comunicación no es una condición suficiente para que esta sea percibida como un problema y que los productores agrícolas actúen en pro de su adaptación. Sin embargo, si éste es comunicado adecuadamente (comprendido) será fundamental para visibilizar sus impactos y coadyuvar al éxito en el diseño e implementación de estrategias de carácter técnico productivo.

En dicha tematización, la semántica como una construcción colectiva juega un papel fundamental, sin su consideración, se limitará la existencia de operaciones comunicativas entre los productores y sus familias y por tanto, impactará en la compresión del significado del cambio climático y sus efectos en la agricultura en el mediano y largo plazo. En consecuencia, esto reducirá la generación de capacidades adaptativas ante el cambio climático y por ende, el diseño e implementación de acciones proactivas para la adaptación de la agricultura del trópico subhúmedo mexicano.

\section{Literatura citada}

Adger, W. N.; Arnell, N. W. and Tompkins, E. L. 2005. Successful adaptation to climate change across scales. Global Enviromental Change. 15(2):77-86.

Altieri, M. y Nicholls, C. 2008. Los impactos del cambio climático sobre las comunidades campesinas y de agricultores tradicionales y sus respuestas adaptativas. Agroecología. 3:728. https://revistas.um.es/agroecologia/article/view/95471.

Baraldi, C. 1996. Semántica. Glosario sobre la teoría social de Niklas Luhmann. In: G. Corsi, Espósito, E. y Baraldi, C. (Ed.). México, DF. Universidad IberoamericanaITESO/Anthropos. 143-145 pp.

Barrasa, S. 2017. Percepción del cambio climático en comunidades campesinas de la Reserva de la Biosfera La Encrucijada, Chiapas, México. 44-65 pp. 
Boon, H. 2014. Climate change ignorance: an unacceptable legacy. The Australian Educational Researcher. 42(4):405-428.

Buys, L.; Miller, E. and Van Megen, K. 2012. Conceptualising climate change in rural Australia: Community perceptions, attitudes and (in) actions. Regional Environmental Change. 12(1):237-248.

Cagle, L. E. and Tillery, D. 2015. Climate change research across disciplines: the value and uses of multidisciplinary research reviews for technical communication. Technical Communication Quaterly. 24(2):147-163.

Campos, M.; Herrador, D. y Michael, C. M. 2013. Estrategias de adaptación al cambio climático en dos comunidades rurales de México y El Salvador. Boletín de la Sociedad de Geçografos españoles. 61:329-349.

Casanova-Pérez, L. 2015. Transformación de los agroecosistemas en el trópico subhúmedo veracruzano ante el cambio climático: un enfoque autopoiético. Tesis doctoral. Colegio de Postgraduados. $220 \mathrm{p}$.

Casanova-Pérez, L.; Martínez-Dávila, J. P.; López-Ortiz, S.; Landeros-Sánchez, C. and LópezRomero, G. 2016. Sociocultural dimension in agriculture adaptation to climate change. Climatic Change. 40(8):848-862.

Eaking, H.; Wightman, P. M.; Hsu, D.; Vladimir, R.; Fuentes-Contreras, E. and Cox, M. 2014. Information and communication technologies and climate change adaptation in Latin America and the Caribbean: a framework for action. Climate and Development. 7(3):208-222.

ENDUTIH. 2019. Encuesta sobre disponibilidad y uso de tecnologías de la información y la comunicación en los hogares de México. Comunicado de prensa 179/19. https://www.inegi.org.mx/contenidos/saladeprensa/boletines/2019/otrtemecon/endutih_ 2018.pdf.

Eskjær, M. 2012. The Regional dimension: how regional media systems condition global climatechange communication. J. Inter. Interc. Comm. 6(1):61-81.

Fernández-Llamazares, Á.; Méndez-López, M. E.; Díaz-Reviriego, I.; McBride, M. F.; Phyhälä, A.; Rosell-Mele, A. and Reyes-García, V. 2015. Links between media communication and local perceptions of climate change in an indigenous society. Climatic Change. 131(2):307-320.

Fowler, C. 2008. Crop diversity: neolithic foundations for agriculture's future adaptation to climate change. Ambio. J. Human Environ. 37(14):498-501.

González, E. J. 2012. La representación social del cambio climático: una revisión internacional. Rev. Mex. Investig. Educ. 17(55):1035-1062.

Howe, P. D.; Markowitz, E. M.; Ming Lee, T.; Ying, K. C. and Leiserowitz, A. 2013. The role of culture and traditional knowledge in climate change adaptation: insights from East Kimberley, Australia. Nature Climate Change. 23(3):352-356.

Ibarra, A. M. 2001. La investigación en comunicación masiva y comportamiento social: una visión de su historia y concepción. Comunicación y Sociedad. 40:11-64. http://www.cucsh1.udg.mx/publicacionesite/pperiod/comsoc/pdf/40_2001/11-64.pdf.

INEGI. 2010. Manual de cartografía. Censo 2010. https:/www.inegi.org.mx/geo/contenidos/ geoestadistica/doc/manual_cartografia_censal.pdf.

IPCC. 2013. Cambio climático 2013. Base de ciencia física. Afirmaciones principales del resumen para responsables de políticas. http://www.ipcc.ch/news_and_events/docs/ar5/ar5_wg1_ headlines_es.pdf. 
Lata, S. and Numm, P. 2012. Mispercetions of climate change-risks as barriers to climate change adaptation: a case of study from the Rewa Delta, Fidji. Climatic Change. 110(1):169-186.

Lavid, J. 2003. Semántica y discurso. Rev. Española de Lingüística. 33(2):345-376.

Leal-Filho, W. 2009. Communicating climate change: challenges ahead and action needed. Inter. J. Climate Change Strategies and Management. 1(1):6-18.

Leombruni, L. V. 2015. How you talk about climate change matters: a communication network perspective on epistemic skepticism and belief strength. Global Environmental Change. 35:148-161. https://doi.org/10.1016/j.gloenvcha.2015.08.006.

Maeseele, P. and Pepermans, Y. 2017. Climate change communication and ideology. Oxford Research Encyclopedia of Climate Science. Doi: 10.1093/acrefore/ 9780190228620.013.578.

McNaught, R.; Warrick, O. and Cooper, A. 2014. Communicating climate change for adaptation in rural communities: a Pacific study. Regional. Environmental Change. 14(4):1491-1503.

Moser, S. and Dilling, L. 2004. Making climate hot.Communicating the urgency and challenge of global climate change. Environment. 46(10):32-46.

Olmos, E.; González, M. E. y Contreras, M. R. 2013. Percepción de la población frente al cambio climático en áreas naturales protegidas de Baja California Sur, México. 35:1-21. https://journals.openedition.org/polis/9158.

O’Neill, S. J.; Boykoff, M.; Neemeyer, S. and Day, S. A. 2013. On the use of imagery for climate change engagement. Global Environmental Change. 23(2):413-421.

Newig, J. 2011. Climate change as an element of sustainability communication. Sustainability Communication. Godemann, J. and Michelsen, G. Springer Netherlands. 119-128 pp.

Nerlich, B.; Koteyko, N. and Brown, B. 2010. Theory and language of climate change communication. Wiley Interdisciplinary Reviews. Climate Change. 1(1):97-110.

Nielsen, J. Ø. and Reenberg, A. 2010. Cultural barriers to climate change adaptation: a case study from northern Burkina Faso. Global Environmental Change. 20(1):142-152.

Ockwell, D.; Whitmarsh, L, and O'Neill, S. 2009. Reorienting climate change communication for effective mitigation forcing people to be green or fostering grass-roots engagement? Science Communication. 30(3):305-327.

Osorio, F. 2011. La evolución de los mass media. Revista Mad - Universidad de Chile. 25: 43-50. http://www.facso.uchile.cl/publicaciones/mad/25/osorio03.pdf.

Pignuoli, S. 2013. El modelo sintético de comunicación de Niklas Luhmann. Cinta Moebio. 47:59-73. http://doi.org/10.4067/S0717-554X2013000200001.

Rahman, M. I. 2013. Climate change: a theoretical review. Interdisciplinary Description of Complex Systems. 11(1):1-13.

Raymond, C. M. and Spoehr, J. 2013. The acceptability of climate change in agricultural communities: comparing responses across variability and change. J. Environ. Manag. 115(30): 69-77. http://doi: 10.1016/j.jenvman.2012.11.003.

Safi, A. S.; Smith, J. W. and Liu, Z. 2012. Rural Nevada and climate change: vulnerability, beliefs and risk perception. Risk Analysis. 32(6):1041-1059.

Sagástegui, D. 2015. Comunicación, cultura científica y tecnológica: transformaciones conceptuales y contextuales. In: Herrera, S.; Orozco, C. E. y Quijano, E. (Coord.). Comunicar Ciencia en México. México DF. ITESO. 23-56 pp.

Soares, D. y García, A. 2014. Percepciones campesinas indígenas acerca del cambio climático en la cuenca de Jovel, Chiapas-México. Cuadernos de Antropología Social. 39:63-89. http://dx.doi.org/10.34096\%2Fcas.i39.1286. 
Solis, R. y Salvatierra, B. 2013. Percepción social del cambio climático en áreas destinadas voluntariamente a la conservación en comunidades indígenas de Oaxaca y Chiapas. Rev. Cient. Inv. Reg. 35(1):29-53.

Schweizer, S.; Davis, S. and Thompson, J. L. 2013. Changing the conversation about climate change: a theoretical framework for place-based climate change engagement. Environmental Communication. 7(1):42-62.

Sterman, J. D. 2011. Communicating climate change risks in a skeptical world. Climate Change. 108:811-826. https://link.springer.com/article/10.1007/s10584-011-0189-3.

Wibeck, V. 2013. Enhancing learning, communication and public engagement about climate change-some lessons from recent literature. Environmental Education Research. 20(3):387-411. https://doi.org/10.1080/13504622.2013.812720.

Weingart, P.; Engels, A. and Pansegrau, P. 2000. Risks of communication: discourses on climate change in science, politics, and the mass media. Public Understanding of Science. 9(3):261-278.

Wolf, J. and Moser, S. 2011. Individual understandings, perceptions, and engagement with climate change: insights from in-depth studies across the world. Wire's Climate Change. 2(4):547-589. 